\title{
An Insight in to Various Metallic Oxide Nanoparticles as Antimicrobials and Their Applications in Dentistry
}

\author{
Hema Kanathila ${ }^{1}$, Ashwin M. Pangi², Suvidha Patil ${ }^{3}$, Shilpa Shirlal ${ }^{4}$, Rahul Jaiswal ${ }^{5}$ \\ 1,3,5 Department of Prosthodontics, KAHER's KLE VK Institute of Dental Sciences, Belagavi, Karnataka, \\ India, ${ }^{2}$ Department of Prosthodontist, Aesthetix Dental Clinic, Belagavi, Karnataka, India, ${ }^{4}$ Department \\ of Prosthodontics, Century International Institute of Dental Sciences, Kasargod, Kerala, India.
}

\section{ABSTRACT}

Oral cavity contains numerous microorganisms. Among them, some are pathogenic and cause infections. Hence to control and prevent such infections, a lot of research has been conducted on different materials using various advanced techniques. Nanotechnology and nanoscience have emerged in the recent years exploring the antimicrobial effects of metal nanoparticles. Metal oxide nanoparticles have shown good results against microorganisms.

Distinct physical, chemical and biologic properties of metallic oxide nanoparticles make them efficient antimicrobial agents. Both the size and high surface to volume ratio have been considered as the reason for their microbicidal efficacy. Metallic oxide nanoparticles show high durability and less cell toxicity compared to organic nanoparticles. And this positive response of metallic oxide nanoparticles make their use in medical and dental fields more promising.

Most of the infections of oral cavity are fungal, bacterial or viral. In dentistry, microorganisms are believed to cause failures of many treatment by causing infections. To achieve a long-term success of treatment rendered, various possibilities have been studied. Application of this nanotechnology in dentistry termed as nano dentistry, has brought many effective changes in the control of oral infections as well as changes in the dental materials.

Various metallic nanoparticles like silver nanoparticles, zinc oxide nanoparticles etc., have been used as well as modified in order to apply in dentistry. Many researches have been carried out by incorporating metallic oxide nanoparticles with dental materials which have shown excellent antimicrobial effectiveness. This review focuses on metal and metallic oxide nanoparticles and their effectiveness as antimicrobials and their various applications in dental field.
Corresponding Author:

Dr. Hema Kanathila,

Department of Prosthodontics,

KAHER's KLE VK Institute of

Dental Sciences, Belagavi,

Karnataka, India.

E-mail: hemak_19@yahoo.com

DOI: $10.14260 / j e m d s / 2021 / 572$

How to Cite This Article:

Kanathila H, Pangi AM, Patil S, et al. An insight in to various metallic oxide nanoparticles as antimicrobials and their applications in dentistry. J Evolution Med Dent Sci 2021;10(33):2803-2808, DOI: 10.14260/jemds/2021/572

Submission 07-04-2021,

Peer Review 12-06-2021,

Acceptance 19-06-2021,

Published 16-08-2021.

Copyright (c) 2021 Hema Kanathila et al. This is an open access article distributed under Creative Commons Attribution License [Attribution 4.0 International (CC BY 4.0)]

\section{KEY WORDS}

Nanoparticles, Antimicrobials, Metallic Oxides, Dental Materials, Dentistry 


\section{BACKGROUND}

Due to the growth in the number of microorganisms and their resistance to antimicrobials, scientists have researched on materials that can be, effective against microorganisms as well be cost effective. Decreasing the particle size has been found to be an efficient tool in improving the biocompatibility. Nanoparticles of metals have various applications in industry, agriculture and healthcare. Antimicrobials in the form of nanoparticles have been studied, and suggested their use as bactericidal materials. In various research studies, metals and metallic oxides have proved to show antimicrobial activity at low concentrations. 1,2,3

The antimicrobial activity of different metals like silver $(\mathrm{Ag})$, gold $(\mathrm{Au})$, titanium (Ti), copper $(\mathrm{Cu})$ and zinc $(\mathrm{Zn})$, which are considered to be safe for human beings, have been used in various areas since a long time. ${ }^{4}$ As years passed, metallic oxide nanoparticles such as silver oxide (Ag20), titanium dioxide ( $\mathrm{TiO} 2)$, zinc oxide $(\mathrm{ZnO})$, calcium oxide ( $\mathrm{CaO}$ ), magnesium oxide $(\mathrm{MgO})$ and copper oxide $(\mathrm{CuO})$ were identified to display antimicrobial activity.

Oral cavity has numerous microorganisms which can cause various infections. Dental diseases have multiple etiological factors, but the most common cause is bacteria. Oral infections can influence the progression and pathogenesis of many systemic diseases. Hence maintaining oral health is a major goal in dentistry. Nanomaterials along with nanotechnology in dentistry help in bringing down the microbial infections.

The accurate mechanism of action of nanometals is still being studied. The process thought to be behind its antimicrobial activity is that they act by deactivating the cellular enzyme and DNA by engaging the electron donating groups such as carboxylates, Amides, Thiols, Indoles, Hydroxyls, etc. They can act by creating pores in bacterial cell walls which can lead to increased permeability and cell death. Certain possibilities proposed in this regard include free metal ion toxicity due to the dissolution of metals from the surface of nanoparticles or oxidative stress caused by generating reactive oxygen species (ROS) on the surfaces of nanoparticles. ${ }^{5}$

Antimicrobial effectiveness depends on the kind of the materials used for preparing the nanoparticles and their particle size.6,7 Decreasing the particle size of the materials is considered to be an efficient tool for improving biocompatibility. ${ }^{5}$ Nanotechnology at present gives a good platform for changing the physicochemical properties of various metals to transform them into effective antimicrobials. Hence antimicrobial efficacy of various nanometals owe to their morphology and physicochemical properties. ${ }^{6,8}$

The antimicrobial efficacy of nanoparticles is found to be greatly influenced by its shape.9,10 Nanoparticles when compared to their own larger particles show different characteristic features. This can be credited to the fact that the surface/volume ratio of the nanoparticles increase with the reduction of the particle size. ${ }^{11,12}$ Because of this small particle size, nanoparticles have a better penetration into cells and tissues, showing strong bactericidal effect. $13,14,15$ It is believed that, the positively charged surface of the metallic nanoparticles help in their attachment to the negatively charged bacterial cell wall surface thus resulting in a higher bactericidal effect. 6

\section{NANOMETALS AS ANTIMICROBIALS} IN DENTISTRY

Various studies done on the below-mentioned nanoparticles have been applied into different fields of dentistry starting from the biomaterial aspect to the treatment level considerations. Silver, titanium dioxide and zinc oxide nanoparticles have been broadly studied and applied in various dental materials

\section{Silver (AG) and Silver Oxide Nanoparticles (AG 20)}

Silver nanoparticles are employed as antimicrobial agents in numerous products and have vast biomedical applications. Among nanometals, with antimicrobial properties, silver is being used against microbial infections since many years. The antibacterial efficacy of silver nanoparticles reduce patient infection, antibiotic dependence as well as associated costs. Besinis et al. in their study concluded that the antibacterial effectiveness of Ag nanoparticles on S. Mutans was high-up than that of chlorhexidine. ${ }^{16}$

Hernández-Sierra et al. in their comparative study on the antimicrobial action of $\mathrm{Ag}$ nanoparticles, $\mathrm{ZnO}$, and $\mathrm{Au}$ against Streptococcus Mutans (S. Mutans) showed that Ag exhibited the highest activity against $S$. Mutans. They also suggested that as S. Mutans are being responsible for dental caries, Ag nanoparticles may be considered helpful. ${ }^{17}$ Properties based on the shape of nanoparticles have also been studied by many researchers. Bera et al. stated that the size as well as the shape of the fluorescent Ag nanoparticles $(1-5 \mathrm{~nm})$ controlled their antimicrobial activity against Bacillus Megaterium, Staphylococcus Epidermidis and Pseudomonas aeruginosa.

Ag20 nanoparticles were found to have good antimicrobial activity. Sondi and Salopek-Sondi in their study proved the antimicrobial efficacy of Ag2O nanoparticles against E. coli. ${ }^{18}$

\section{Titanium Dioxide (TiO2) Nanoparticles}

They are considered as advanced materials because of their impressive optical, photo-catalytic and dielectric properties. Titanium dioxide (TiO2) is used as a disinfecting and cleansing material. It is used for environmental purification. ${ }^{19}$ These properties are very well utilized in eliminating bacteria and harmful agents from air and water. ${ }^{20}$ These nanoparticles are also used for sterilizing surfaces in healthcare centres.

Antimicrobial property of $\mathrm{TiO} 2$ is attributed to its size, shape and crystal structure. ${ }^{21}$ The oxidative stress caused by the formation of reactive oxygen species (ROS) may be an important mechanism for TiO2 nanoparticles. The ROS causes site specific DNA damage. ${ }^{22,23}$ Haghighi et al. in their research on the antifungal effect of TiO2 nanoparticles against Candida albicans resistant to fluconazole, showed that the synthesized TiO2 nanoparticles had good antifungal activity on the $C$. albicans resistant to fluconazole. Therefore, it was suggested that TiO2 nanoparticles could be used effectively to inhibit the fungal growth formed on the surface of medical devices. ${ }^{21}$

TiO2 nanoparticles have the potential to generate ROS under UV light. Photocatalytic properties of the $\mathrm{TiO} 2$ nanoparticles make them efficient in eradicating bacteria. But, the use of TiO2 nanoparticles under UV light can cause genetic damage in humans, hence it is restricted. It has been found that, doping of $\mathrm{TiO} 2$ nanoparticles with metal ions can be a 
good idea to solve this problem. ${ }^{24}$ Carré et al. noted that the antibacterial activity was accompanied by lipid peroxidation causing disturbances of the cell integrity. ${ }^{25}$

\section{Zinc Oxide Nanoparticles(Zno)}

Zinc oxide is a bio safe material which exhibits antibacterial properties. Emami-Karvani et al. studied the antimicrobial action of $\mathrm{ZnO}$ nanoparticles against $E$. coli and $S$. aureus bacteria, by using different concentrations and reducing the particle size. ${ }^{26}$ They concluded that the antibacterial action of $\mathrm{ZnO}$ nanoparticles improved by changing the concentrations and by reducing the particle size. This enhanced antibacterial action shown by $\mathrm{ZnO}$ nanoparticles in contrast to its microparticles was attributed to the surface area enhancement. $\mathrm{ZnO}$ nanoparticles exhibit high photocatalytic properties that enhance their antimicrobial efficiency.

Azam et al. in their comparative analysis of antimicrobial activity of $\mathrm{ZnO}, \mathrm{CuO}$, and $\mathrm{Fe} 2 \mathrm{O} 3$ nanoparticles against gram negative and gram-positive bacteria reported $\mathrm{ZnO}$ as the most bactericidal. ${ }^{27} \mathrm{~A}$ few studies have demonstrated that metal ion doped nanoparticles can increase antimicrobial properties of metallic nanoparticles. Another study showed that the titanium doped $\mathrm{ZnO}$ powders showed antimicrobial action against E. coli and S. aureus. ${ }^{28}$

\section{Copper (Cu) and Copper oxide (CuO)} Nanoparticles

Copper nanoparticles have unique biological, chemical and physical properties.24 Mahapatra et al. investigated the antibacterial activity of $\mathrm{CuO}$ nanoparticles and reported that copper oxide nanoparticles showed good antibacterial activity against the tested bacteria. ${ }^{29}$

Azam et al. investigated the antibacterial activities of $\mathrm{CuO}$ nanoparticles against $S$. aureus, Pseudomonas aeruginosa, $B$. subtilis and E. coli. According to their study, nanoparticles exhibited size dependent -inhibitory effects against bacteria. These nanoparticles prevent bacterial growth by entering the nano- pores existing on the cellular membranes of most of the bacteria. ${ }^{30}$

\section{MATERIAL ASPECTS}

\section{Impression Materials}

Dental impressions form vital part of all specialities of dentistry. The most commonly used impression material in dentistry is the hydrocolloid alginate impression material. Impressions get contaminated with saliva as well as blood during clinical procedures and there are chances of cross infection. The incorporation of different antimicrobial agents into impression materials can be effective in decreasing crossinfection.

Alginate powder can be incorporated into water containing silver hydrosol to provide an antimicrobial effect, which will prevent the contamination of stone cast from the infected impression. ${ }^{31,32}$ Wangchuk et al. in their study, evaluated the invitro antimicrobial effectiveness of silver nanoparticles incorporated into alginate against Staphylococcus aureus. According to this study, silver nanoparticles (AgZrPO4) at different concentrations added to the powder of impression material showed inhibitory effect against pathogenic bacteria. ${ }^{33}$

\section{Dental Cements}

They play a key role in restorations as well as cementation of crowns and bridges. So antimicrobial efficacy of dental cements used has become an important criterion, to prevent the pathogenic microorganisms from gaining access into the cavity or the prepared tooth after cementation. The antibacterial activity shown by the luting cements is important considering the microleakage after cementation. Considering this, studies were carried out on the efficacy of nanometals in inhibiting microorganisms. Silver nanoparticles show good antimicrobial activity, which is mainly due to the liberation rate of silver ions. These reactive ions act on the cell wall of the bacteria and result in the structural changes by binding to the tissue protein and causing cell death. Magalhaeces et al. in their study, 34 incorporated silver nanoparticles into glass ionomer and resin cements and the antimicrobial action against $S$. mutans was studied. These cements doped with silver nanoparticles presented significant antimicrobial properties compared to cements without nanoparticles.

Glass ionomer-containing $\mathrm{TiO} 2$ naoparticles possessed antibacterial activity against S.mutans in contrast to the unmodified GIC. ${ }^{35}$ A study evaluating the antibacterial action of zinc oxide nanoparticles incorporated self-cured GIC and light cured resin reinforced GIC on $S$. mutans biofilms, concluded that $\mathrm{ZnO}$ nanoparticles incorporated into GIC, at different concentrations did not help in their antibacterial action. ${ }^{36}$

\section{Dental Composites}

The dental composites are commonly used in dental restorations due to their aesthetic superiority and strong bonding ability and strength. Plaque accumulation on these materials can cause recurrent caries and ultimately failure of restorations. Titanium and silver particles were incorporated into dental composites to provide antimicrobial properties as well as to improve the biocompatibility. To reduce the biofilm accumulation over composite, restorative materials with antimicrobial properties have been developed by the incorporation of silver nanoparticles into composite resins. Cheng et al. reported that silver nanoparticles incorporated into composite resins enabled good mechanical properties along with notable antimicrobial effect, at lower concentrations. ${ }^{37}$

Reham et al. evaluated antimicrobial effectiveness of $\mathrm{ZnO}$ nanoparticles incorporated into resin composites against organisms causing caries and the results showed $85 \%$ reduction in the growth of different kinds of bacteria which was in the study. ${ }^{38}$ In a study conducted by Shahin Kasraei et al. antibacterial properties of resin based composite with zinc oxide and silver nanoparticles concluded that it could inhibit the growth of $S$. mutans and lactobacillus. ${ }^{39}$

Light cured flowable composite can function as an effective antimicrobial by adding silver hydrosol. This silver hydrosol that gets leached from the composite resin matrix can help to decrease the incidence of dental caries. ${ }^{40}$ Such studies showing antimicrobial properties of silver nanoparticles-containing composite resins might reduce the development of secondary 
or recurrent caries by reducing the bacterial biofilm formation on the teeth and thereby increasing the longevity of the restorations.

\section{Endodontic Materials}

\section{Guttapercha}

Success of endodontic care is connected to the bacterial elimination, to a large extent. Out of many that have been used as root canal filling materials, gutta-percha is the most widely used. The zinc oxide component present in gutta-percha makes it have its own antimicrobial property. Iranian researchers, Dianat and Ataie had introduced nanosilvergutta-percha, to enhance the antibacterial effectiveness of gutta-percha. The new material demonstrated significant antimicrobial effect against Enterococcus faecalis, Candida albicans, Staphylococcus aureus and Escherichia coli. ${ }^{41}$

\section{Mineral Trioxide Aggregate (MTA)}

MTA is a well-known dental root repair material. It is used for an apical plug formation during apexification, in case of any root perforations during root canal treatment and in cases of internal root resorption. Also used as root-end filling material and Pulp capping material. Samiei et al. modified MTA by incorporating silver nanoparticles and it was found to act against oral bacteria and fungi species. They concluded that a group-containing MTA possesses higher antimicrobial action compared to unmodified MTA. ${ }^{2}$

Even though AgNP is a potent antimicrobial, there are only a few studies incorporating it in endodontic materials.

\section{Dental Adhesives}

Dental adhesives are materials which help in the adhesion and cohesion of two different materials or between material and natural teeth. Li et al. in their study incorporated silver nanoparticles (AgNPs) to an adhesive system inorder to evaluate the bacterial inhibition. According to the results of this study, AgNPs reduced colony forming units (CFU) number and lactic acid production on biofilms suggesting their antibacterial potential. 43

Melo et al. in their study evaluated antimicrobial efficacy by incorporating silver nanoparticles to an adhesive system and results showed a reduced metabolic activity on biofilm, compared to control group without AgNPs. ${ }^{44}$ Mahshid S et al. studied the effect of adding zinc oxide nanoparticles to dental adhesives on their antimicrobial activity and bond strength. According to their results, addition of zinc oxide nanoparticles to dental adhesives inhibited the formation of Streptococcus mutans colonies, without compromising the bond strength. 45

\section{Acrylic Resins}

Dentures are fabricated by poly methyl methacrylate (PMMA) acrylic resin. Various factors can contribute to the roughness of their inner surface which can act as a niche for microbial (candida species) colonization, which can cause denture stomatitis in denture wearers. ${ }^{46}$ Due to the smaller size, silver nanoparticles possess greater dispersion in PMMA matrix and produce larger area for oxidation. The release of silver ions acts by rupturing the cell wall and denaturating the protein and ultimately leading to microbial death, thus explaining the antimicrobial action and effectiveness of silver nanoparticles.
Acosta-Torres et al. in their research observed that PMMAAgNPs specimens showed less adherence of Candida albicans compared to unmodified PMMA, thus reporting the antifungal potential of AgNPs incorporated into acrylic resin. ${ }^{47}$

\section{Tissue Conditioners or Soft Liners}

Tissue conditioners are used in denture patients who present with inflamed or abused mucosa and getting them back to normal health. It is mainly used to aid in the treatment of chronic soreness caused by dentures. Tissue conditioners are susceptible to microbial colonization. Many studies were conducted by addition of metallic and metallic oxides to tissue conditioners to study their antimicrobial activity. Nam in his study, incorporated AgNPs into a commercial tissue conditioner, in different concentrations. Their inhibitory effect was evaluated against Staphylococcus aureus, Streptococcus mutans, and Candida albicans. He reported that the modified tissue conditioner presented antimicrobial properties even at lower concentrations.

\section{Implants}

Dental implants have become an integral part of reconstructive dentistry. Bacterial invasion can occur during surgery or post operatively. Infection being one of the most important complications in Implantology, various measures have been considered inorder to avoid bacterial contamination. It has been reported in various literatures that titanium dioxide nanoparticles coating on titanium implants can increase antimicrobial efficacy of implants. Singaravel et al. in their study, evaluated the antifungal effect of titanium, zirconium and aluminium nanoparticles coated titanium oxide plates. Titanium oxide nanoparticles coated titanium plates showed greater antifungal effect against Candida albicans, compared to zirconium oxide and aluminium oxide. 48

Flores et al. suggested that incorporation of silver nanoparticles into titanium implants as a method to guard implant surface against the pathogen. And they also reported that silver nanoparticles showed bactericidal action at lesser concentration which does not interfere with the osseointegration process. ${ }^{49}$

Zhao et al. in their study, incorporated silver nanoparticles into titania nanotubes (TiO2 - NTs) on Ti implants. The antibacterial efficacy against Staphylococcus aureus was evaluated. According to this study, Ti implants incorporated with silver nanoparticles showed its ability to prevent bacterial adhesion for a time period of 1 month. ${ }^{50}$

Zhang et al. in their study coated TiO2 implants by silver nanoparticles using Micro Arc Oxidation(MAO) and their results showed enhanced antimicrobial activity which was attributed to the interation between silver nanocrystals and bacterial cell membrane. ${ }^{51} \mathrm{~A}$ study conducted by Xiaojing He et al. suggested that the micro arc oxidation (MAO) technique effectively introduces inorganic antibacterial metals (eg: Ag, $\mathrm{Cu}, \mathrm{Zn}$ ) into biomedical implants, thus successfully solving the issue of periimplant infection. Flores et al. studied the antibacterial activity of silver nanoparticles (AgNPs) against Pseudomonas aeruginosa and reported that the incorporation of AgNPs into Ti implants as an efficient approach to guard the surface of implants against microbial colonization. ${ }^{52}$

Miguel A et al. studied antimicrobial activity of metal oxide nanoparticles against pathogens causing periimplantitis. In 
their study they considered six metal $(\mathrm{Ag})$ and metal oxides [cuprous oxide $(\mathrm{Cu} 2 \mathrm{O})$, cupric oxide $(\mathrm{CuO})$, zinc oxide $(\mathrm{ZnO})$, titanium dioxide (TiO2), tungsten oxide (WO3)] and two of their composites[( $\mathrm{Ag}+\mathrm{CuO}),(\mathrm{Ag}+\mathrm{ZnO})]$ against bacterial pathogens. The antimicrobial activity of the nanoparticles tested had silver showing the most and tungsten oxide the least antimicrobial effect. And they suggested that composites of nanoparticles should also be considered as antimicrobial agents. In this study, bacteriostatic activity showed by the composites were greater than that of nanoparticles alone. 53

\section{Maxillofacial Prosthesis}

Maxillofacial prostheses are more prone for contamination and thereby can cause infections. Maxillofacial prostheses fabricated from silicone materials are more prone to fungal infections caused by Candida albicans. Coating silicone materials with silver nanoparticles could be advantageous to the patients using maxillofacial prostheses, to prevent or reduce fungal infection. Silver nanoparticles have been incorporated into maxillofacial prosthesis and it showed reduced adherence of Candida albicans to maxillofacial prosthesis surface. ${ }^{54}$

\section{CONCLUSIONS}

Many microorganisms and antimicrobial resistant microorganisms pose a real threat to human life. Advances in the field of nano-structures and nano-materials with suitable properties led to an increase in the making of stable nanoparticles that are capable of biomedical applications. Applications of metal and metallic oxide nanoparticles, and their surface modifications, with good antimicrobial activity in lesser concentration locally destroy pathogens, without being toxic. Hence metallic oxide nanoparticles might be considered as a new alternative to most of the antibiotics and if applied in drug delivery systems in medical and dental field can be a boon to the human race, where antiobiotic resistant pathogens have grown at an alarming rate.

Financial or other competing interests: None.

Disclosure forms provided by the authors are available with the full text of this article at jemds.com.

\section{REFERENCES}

[1] Liu Y, He L, Mustapha A, et al. Antibacterial activities of zinc oxide nanoparticles against Escherichia coli 0157:H7. J Appl Microbiol 2009;107(4):1193-201.

[2] Raghupathi KR, Koodali RT, Manna AC. Size-dependent bacterial growth inhibition and mechanism of antibacterial activity of zinc oxide nanoparticles. Langmuir 2011;27(7):4020-8.

[3] Panacek A, Kolar M, Vecerova R, et al. Antifungal activity of silver nanoparticles against Candida spp. Biomaterials 2009;30(31):6333-40.

[4] Malarkodi C, Rajeshkumar S, Paulkumar K, et al. Biosynthesis and antimicrobial activity of semiconductor nanoparticles against oral pathogens. Bioinorg Chem Appl 2014;2014:347167.
[5] Kim JS, Kuk E, Yu KN, et al. Antimicrobial effects of silver nanoparticles. Nanomedicine 2007;3(1):95-101.

[6] Seil JT, Webster TJ. Antimicrobial applications of nanotechnology: methods and literature. Int J Nanomedicine 2012;7:2767-81.

[7] Adibkia K, Alaei-Beirami M, Barzegar-Jalali M, et al. Evaluation and optimization of factors affecting novel diclofenac sodium-eudragit RS100 nanoparticles. Afr J Pharm Pharmacol 2012;6(12):941-7.

[8] Mohammadi G, Valizadeh H, Barzegar-Jalali $M$, et al. Development of azithromycin-PLGA nanoparticles: physicochemical characterization and antibacterial effect against Salmonella typhi. Colloids Surf B Biointerfaces 2010;80(1):34-9.

[9] Pal S, Tak YK, Song JM. Does the antibacterial activity of silver nanoparticles depend on the shape of the nanoparticle? A study of the Gram-negative bacterium Escherichia coli. Appl Environ Microbiol 2007;73(6):1712-20.

[10] Bera RK, Mandal SM, Raj CR. Antimicrobial activity of fluorescent ag nanoparticles. Lett Appl Microbiol 2014;58(6):520-6.

[11] Buzea C, Pacheco II, Robbie K. Nanomaterials and nanoparticles: sources and toxicity. Biointerphases 2007;2(4):MR17-71.

[12] Adibkia K, Barzegar-Jalali M, Nokhodchi A, et al. A review on the methods of preparation of pharmaceutical nanoparticles. J Pharm Sci 2010;15:303-14.

[13] Niederberger M, Pinna N. Metal oxide nanoparticles in organic solvents. USA: Springer 2009.

[14] Fellahi O, Sarma RK, Das MR, et al. The antimicrobial effect of silicon nanowires decorated with silver and copper nanoparticles. Nanotechnology 2013;24(49):495101.

[15] Mohammadi G, Nokhodchi A, Barzegar-Jalali M, et al. Physicochemical and anti-bacterial performance characterization of clarithromycin nanoparticles as colloidal drug delivery system. Colloids Surf B Biointerfaces 2011;88(1):39-44.

[16] Besinis A, De Peralta T, Handy RD. The antibacterial effects of silver, titaniumdioxide and silica dioxide nanoparticles compared to the dental disinfectant chlorhexidine on streptococcus mutans using a suite of bioassays. Nanotoxicology 2014;8(1):1-16.

[17] Hernández-Sierra JF, Ruiz F, Pena DCC, et al. The antimicrobial sensitivity of Streptococcus mutans to nanoparticles of silver, zinc oxide and gold. Nanomedicine 2008;4(3):237-40.

[18] Sondi I, Salopek-Sondi B. Silver nanoparticles as antimicrobial agent: a case study on E. coli as amodel for Gram-negative bacteria. J Colloid Interface Sci 2004;275(1):177-82.

[19] Fujishima K, Honda K. Electrochemical photolysis of water at a semiconductor electrode. Nature 1972;238(5358):37-8.

[20] Tojo S, Tachikawa T, Fujitsuka M, et al. The photoabsorption and surface feature of nano-structured TiO2 coatings. J Phys Chem C 2008;112(38):14948-54.

[21] Haghighi F, Mohammadi SR, Mohammadi P, et al. Antifungal activity of TiO2 nanoparticles and EDTA on Candida albicans biofilms. Infect Epidemiol Med 2013;1(1):33-8. 
[22] Cioffi N, Rai M. Nano-antimicrobials. Synthesis and characterization of novel nano antimicrobials. Berlin Heidelberg: Springer 2012.

[23] Roy AS, Parveen A, Koppalkar AR, et al. Effect of nanotitanium dioxide with different antibiotics against methicillin-resistant staphylococcus aureus. J Biomater Nanobiotechnol 2010;1(1):37-41.

[24] Dizaj SM, Lotfipour F, Barzegar-Jalali $M$, et al. Antimicrobial activity of the metals and metal oxide nanoparticles. Mater Sci Eng C Mater Biol Appl 2014;44:278-84.

[25] Carré G, Hamon E, Ennahar S, et al. TiO2 photocatalysis damages lipids and proteins in Escherichia coli. Appl Environ Microbiol 2014;80(8):2573-81.

[26] Emami-Karvani Z, Chehrazi P. Antibacterial activity of ZnO nanoparticle on Gram positive and gram-negative bacteria. Afr J Microbiol Res 2011;5(12):1368-73.

[27] Azam A, Ahmed AS, Oves M, et al. Antimicrobial activity of metal oxide nanoparticles against Gram-positive and Gram-negative bacteria: a comparative study. Int J Nanomedicine 2011;7:6003-9.

[28] Sun T, Hao H, Hao WT, et al. Preparation and antibacterial properties of titanium-doped $\mathrm{ZnO}$ from different zinc salts. Nanoscale Res Lett 2014;9(1):98.

[29] Mahapatra O, Bhagat M, Gopalakrishnan C, et al. Ultrafine dispersed $\mathrm{CuO}$ nanoparticles and their antibacterial activity. J Exp Nanosci 2008;3(3):185-93.

[30] Azam A, Ahmed AS, Oves M, et al. Size-dependent antimicrobial properties of $\mathrm{CuO}$ nanoparticles against Gram-positive and negative bacterial strains. Int J Nanomedicine 2012;7:3527-35.

[31] Li Z, Lee D, Sheng X, et al. Two-level antibacterial coating with both release-killing and contact-killing capabilities. Langmuir 2006;22(24):9820-3.

[32] Hamouda IM. Current perspectives of nanoparticles in medical and dental biomaterials. J Biomed Res 2012;26(3):143-51.

[33] Wangchuk NP, Nisalak P, Thaweboon B, et al. Antimicrobial property of hydrocolloid impression material incorporated with silver nanoparticles against staphylococcus aureus. Matec Web of Conferences 2017;95:01001.

[34] Magalhaeces APR, Santos LB, Lopes LC, et al. Nanosilver application in dental cements. International Scholarly Research Notices 2012:2012:365438.

[35] Elaska SE, Hamouda IM, Swain MV. Titanium dioide nanoparticles addition to a conventional glass ionomer restorative: influence on physical and antibacterial properties. J Dent 2011;39(9):589-98.

[36] Garcia PPNS, Cardia MFB, Francisconi RS, et al. Antibacterial activity of glass ionomer cement modified by zinc oxide nanoparticles. Microsc Res Tech 2017;80(5):456-61.

[37] Cheng L, Weir MD, Hu HHK, et al. Effect of amorphous calcium phosphate and silver nanocomposites on dental plaque microcosm biofilms. J Biomed Mater Res B Appl Biomater 2012;100(5):1378-86.

[38] Al-Mosawi RM, Al-Badr RM. The study effects of dental composite resin as antibacterial agent which contain nanoparticles of zinc oxide on the bacteria associated with oral infection. IOSR Journal of Dental and Medical Sciences 2017;16(1):49-55.

[39] Kasraei S, Sami L, Hendi S, et al. Antibacterial properties of composite resins incorporating silver and zinc oide nanoparticles on Streptococcus mutans and Lactobacillus. Restor Dent Endod 2014;39(2):109-14.

[40] Kassaee MZ, Akhavan A, Sheikh N, et al. Antibacterial effects of a new dental acrylic resin containing silver nanoparticles. J Appl Polym Sci 208;110(3):1699-3.

[41] Dianat 0, Ataie M. Gutta-percha coated with nano silver particles. Invention Registered 2008:56019.

[42] Samiei M, Aghazadeh M, Lotfi M, et al. Antimicrobial efficacy of mineral trioxide aggregate with and without silver nanoparticles. Iran Endod J 2013;8(4):166-70.

[43] Li F, Weir MD, Chen J, et al. Comparison of quaternary ammonium-containing with nano-silver-containing adhesive in antibacterial properties and cytotoxicity. Dent Mater 2013;29(4):450-61.

[44] Melo MAS, Cheng L, Zhang K, et al. Novel dental adhesives containing nanoparticles of silver and amorphous calcium phosphate. Dent Mater 2013;29(2):199-210.

[45] Saffarpour M, Rahmani M, Tahriri M, et al. Antimicrobial and bond strength properties of a dental adhesive containing zinc oxide nanoparticles. Braz J Oral Sci 2016;15(1):66-9.

[46] Correa JM, Mori M, Sanches HL, et al. Silver nanoparticles in dental biomaterials. Int J Biomater 2015;2015:485275.

[47] Acosta-Torres LS, Mendieta I, Nũnez-Anita RE, et al. Cytocompatible antifungal acrylic resin containing silver nanoparticles for dentures. Int J Nanomedicine 2012;7:4777-86.

[48] Chidambaranathan AS, Mohandas K, Balasubramaniam MK. Comparative evaluation of antifungal effect of titanium, zirconium and aluminium nanoparticles coated titanium plates against Candida albicans. JCDR 2016;10(1):ZC56-9.

[49] Flores CY, Diaz C, Rubert A, et al. Spontaneous adsorption of silver nanoparticles on Ti/TiO2 surfaces. Antibacterial effect on pseudomonas aeruginosa. J Colloid Interface Sci 2010;350(2):402-8.

[50] Zhao L, Wang H, Huo K, et al. Antibacterial nanostructured titania coating incorporated with silver nanoparticles. Biomaterials 2011;32(24):5706-16.

[51] Zhang P, Zhang Z, Li W. Antibacterial coating incorporating silver nanoparticles by micro arc oxidation and ion implantation. Journal of Nanomaterials 2013;2013:542878.

[52] He X, Zhang X, Wang X, et al. Review of antibacterial activity of titanium based implants'surfaces fabricated by micro arc oxidation. Coatings 2017;7(3):45.

[53] Vargas-Reus MA, Memarzadeh K, Huang J, et al. Antimicrobial activity of nanoparticulate metal oxides against peri-implantitis pathogens. Int J Antimicrob Agents 2012;40(2):135-9.

[54] Meran Z. The use of silver nanoparticle as an antifungal coating on silicone facial prosthesis. School of Biomed and Biological Science 2013. 